\title{
Nadadores medalhistas olímpicos: contexto do desenvolvimento brasileiro
}

\author{
Renato Melo Ferreira \\ Eduardo Macedo Penna \\ Varley Teoldo da Costa \\ Luiz Carlos Couto de Albuquerque Moraes
}

\section{Escola de Educação Física, Fisioterapia e Terapia Ocupacional, Centro de Excelência Esportiva (CENESP) e Laboratório de Psicologia do Esporte (LAPES) da Universidade Federal de Minas Gerais, Belo Horizonte, MG, Brasil}

\begin{abstract}
Resumo: Expertise é um campo de conhecimento que estuda as diferentes competências dos experts em seu campo de proficiências, como no esporte. Diversos modelos, a partir da perspectiva ambiental, avaliam fatores que influenciam no desenvolvimento expert. $O$ objetivo deste trabalho foi verificar o contexto do desenvolvimento expert dos nadadores medalhistas olímpicos brasileiros. Oito nadadores que conquistaram, no mínimo, uma medalha em qualquer edição dos Jogos Olímpicos, foram os entrevistados. Realizou-se a transcrição das entrevistas e, posteriormente, uma análise qualitativa foi feita, por meio de mini-unidades (MUs). 218 MUs obedeceram à seguinte classificação: Início da carreira esportiva, presença dos treinadores, influência da família / amigos e prática deliberada. Concluiu-se, a partir dos resultados apresentados, que o contexto do desenvolvimento contribuiu de forma fundamental para a evolução dos atletas. As diversas realidades apontam que não existe necessariamente um padrão restrito de excelência.
\end{abstract}

Palavras-chave: Natação. Atletas. Pesquisa Qualitativa.

\section{Olympic Medal-winning swimmers: The Brazilian context of development}

\begin{abstract}
Expertise as a field is one that studies the authenticity of the expert, meaning the skill or knowledge level he or she has in a certain field, such as athletics. Different models, from the ambient perspective, judge factors that influence the development of the expert. The aim of this study was to verify the context in which Brazilian swimmers develop, specifically those who have won an olympic medal. Eight swimmers who have won at least one medal in any category of the Olympic games were interviewed. The transcripts of the interviews were recorded, after which a qualitative analysis using Meaning - Units (MUs). 218 MUs were classified as follows: when the athlete's career began, the presence of coaches, the influence of family and friends, and deliberated practice. From the results, it can be concluded that the context of development contributed fundamentally to the evolution of the athletes. The diverse realities show that there is not a necessary pattern needed to restrict excellency.
\end{abstract}

Keywords: Swimming. Athletes. Qualitative Research.

\section{Introdução}

Indivíduos com desempenhos excepcionais são reconhecidos por suas vitórias. No entanto, ao avaliar apenas o resultado final de um atleta, e não a sua trajetória desde o primeiro dia de prática esportiva, pode-se cometer uma interpretação erronia deste desempenho. Dessa forma, compreender os fatores que norteiam atletas experts é de fundamental importância para a compreensão da excelência no esporte.

Para se compreender o contexto no qual os melhores indivíduos de qualquer área se desenvolvem, é necessário estabelecer o conceito de expertise. Expertise é o campo de conhecimento de um grupo de expoentes que estuda as diferentes competências dos experts em relação à sua proficiência (SIMON; CHASE, 1973). Em várias áreas de atuação encontram-se tais indivíduos, como na música (LEHMANN; ERICSSON, 1998), na arte (ERICSSON et al., 1993), na enfermagem (ERICSSON; WHYTE; WARD, 2007), no desempenho acadêmico (PLANT et al., 2005) e nos esportes (MORAES; RABELO; SALMELA, 2004 ; FERREIRA, 2010a; 2010b).

Existem duas perspectivas para 0 entendimento do desenvolvimento expert, sendo elas: a genética (nature) e a ambiental (nurture) (SINGER; JANELLE, 1999; BAKER et al., 2003). A perspectiva genética afirma que 0 indivíduo possui uma carga genética que define e destaca as diferenças de uma pessoa da outra, que são fundamentais para se atingir níveis excepcionais de desempenho. Já a perspectiva ambiental argumenta que $o$ ambiente é o principal fornecedor das forças intervenientes para o 
desenvolvimento das competências das pessoas e que elas são consideradas adquiridas.

A partir dessa premissa, a perspectiva ambiental é composta por vários aspectos relevantes ao rendimento de uma pessoa, como os estágios de desenvolvimento (BLOOM, 1985), a presença do treinador (CÔTÉ et al.1995), a relação com a família / amigos (CSIKSZENTMIHALYI et al., 1993; CÔTÉ, 1999; BAKER; HORTON, 2004) e a prática deliberada (ERICSSON et al., 1993).

\section{Estágios de desenvolvimento}

Bloom (1985) realizou um estudo no qual o objetivo foi investigar como $o$ alto desempenho era adquirido e quais forças interferiam no desenvolvimento de jovens expoentes. Os resultados indicaram que a qualidade do apoio recebida desde a infância, por seus pais e treinadores, foi um fator preponderante para 0 progresso de suas carreiras (MORAES; SALMELA; RABELO; VIANA JUNIOR, 2004).

Bloom (1985) sugeriu três estágios: anos iniciais, anos intermediários e anos finais. Nos anos iniciais, os pais são responsáveis por inserirem seus filhos em uma ou mais atividades, proporcionando apoio emocional e financeiro para que não ocorra 0 abandono precoce. Os treinadores assumem o papel de motivadores, que por meio de atividades lúdicas estimulam o prazer da criança em realizar a atividade (CÔTÉ et al., 2009). Os anos intermediários são caracterizados pela prática de apenas uma única atividade, pois os indivíduos atingem níveis mais altos de rendimento, alcançados pela prática, exigindo um aumento de concentração e comprometimento com a atividade (CÔTÉ, 1999; HOLT; DUNN, 2004). Os pais contribuem com o apoio financeiro, como melhores condições de treinamento, o que garante a manutenção da atividade dos filhos em relação à vários aspectos, como os motivacionais (VERNACCHIA et al., 2000) e 0 comprometimento (MALLET; HANRAHAN, 2004).

Nos anos finais, o objetivo é melhoria do rendimento, por meio da procura de treinadores mais capacitados, na tentativa de atingir o mais alto grau de proficiência, assim como por locais que proporcionam melhores condições de prática (SALMELA; MORAES, 2003; MARTINDALE et al., 2005). As famílias se adaptam à vida dos atletas para proporcionar melhores condições de treinamentos. A dedicação, a disciplina, a motivação e a persistência (fatores intrínsecos) são as características fundamentais para o êxito na atividade (MALLET; HANRAHAN, 2004; BENGOECHEA et al., 2004).

\section{Treinador}

Ao avaliarem 17 treinadores experts de

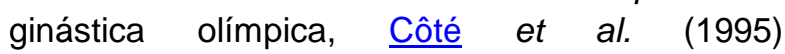
estabeleceram o Modelo do Treinador, que abrange os componentes centrais (organização, treinamento e competição) e os periféricos (características pessoais do atleta, do treinador e os fatores contextuais) do desempenho esportivo.

Dentre os componentes centrais, a organização envolve 0 trabalho com pais e assistentes, o planejamento do treinamento e a ajuda aos atletas com problemas pessoais. $O$ treinamento, altamente deliberado (ERICSSON et al., 1993), centra-se na repetição de determinada técnica ou tática, realizada durante anos (ERICSSON; RORING; NANDAGOPAL, 2007; WARD et al., 2007). Já a competição é o momento que os treinadores utilizam seus conhecimentos, a fim de obterem o máximo resultado. A partir da organização e dos treinamentos, os treinadores estabelecem rotinas para que seus atletas não sintam pressão e/ou estresse que possa provocar 0 abandono da modalidade (GUSTAFSSON et al., 2007).

Nos componentes periféricos, as características pessoais dos atletas são caracterizadas pela motivação, persistência e disciplina (GOULD et al., 1993), pois atletas altamente confiantes e disciplinados conseguem atingir seus objetivos com maior facilidade (HAYS et al., 2007). As características pessoais dos treinadores englobam as fontes de satisfação pessoal, qualidades pessoais e abordagem dada ao treinamento (CÔTÉ et. al, 1995). Os fatores contextuais exemplificam a influência dos pais (CÔTÉ, 1999), das condições financeiras e do papel de treinadores assistentes. A estrutura familiar e financeira influencia diretamente o atleta, pois a falta de condições financeiras pode obrigar a família ou 0 atleta a abandonar 0 ambiente esportivo (MORAES; RABELO; SALMELA, 2004).

Helsen et al. (1998), Feltz et al. (1999), Salmela e Moraes (2003), Ferreira et al. (2010) afirmam que treinadores campeões apresentam como característica principal o encorajamento de suas equipes, além do melhor feedback possível, visando o sucesso durante treinamentos e competições. No entanto, sem a presença dos amigos e da família, se torna mais difícil a manutenção na prática da modalidade esportiva.

\section{Família e amigos}

Ao se analisar o contexto familiar, Côté (1999) investigou a influência da família no desenvolvimento de jovens atletas. Os principais resultados apontam que a família parece assumir 
diversos papéis durante o desenvolvimento da carreira dos filhos. Nos anos iniciais, os pais proporcionam oportunidades para que os filhos tenham a maior diversidade possível de experiências no esporte. Nos anos intermediários, os pais aumentam o interesse pelo esporte que 0 filho escolheu para se especializar. Já nos anos finais, os pais se destacam pelo auxílio ao filho na busca pela melhor estrutura de treinamento.

A participação efetiva, e positiva, dos pais no desenvolvimento da carreira dos filhos ocorre por intermédio do apoio financeiro e motivacional (FALK et al., 2004), sendo uma influência fundamental para o desenvolvimento ao longo da carreira (BAKER; HORTON, 2004; CÔTÉ et al., 2009), possibilitando aos mesmos se tornarem experts (ERICSSON; PRIETULA; COKELY, 2007).

O estudo de Moraes e Sousa (2004) teve como objetivo analisar o envolvimento da família no desenvolvimento de atletas. Os resultados mostraram um envolvimento efetivo dos pais durante os estágios de desenvolvimento. Os pais apoiavam os atletas por meio de recursos financeiros, na busca de melhores condições e na motivação de seus filhos. Tais resultados corroboram os encontrados por Durand-Bush e Salmela (2002) e Moraes e Salmela (2003).

McCarthy e Jones (2007) identificaram em seu estudo que as principais fontes prazerosas para os atletas durante os anos iniciais de desenvolvimento foram o envolvimento social e a relação com os amigos, confirmando, dessa forma, os resultados encontrados por Samulski et. al (2009). A presença de treinadores e familiares é fundamental, porém se o atleta não praticar de forma deliberada, dificilmente atingirá a excelência esportiva.

\section{Prática Deliberada}

Para o atleta ser considerado expert, além do apoio da família, de um ambiente favorável para o desenvolvimento, passando por todos os estágios, e, um treinador capacitado e comprometido com seu trabalho, é necessário aproximadamente 10 anos ou dez mil horas de prática deliberada para atingirem o desempenho excepcional (ERICSSON et al., 1993; ERICSSON; RORING; NANDAGOPAL, 2007; WARD et al., 2007; COLVIN, 2007; WILLIAMS; ERICSSON, 2008).

Prática deliberada pode ser conceituada como uma atividade altamente estruturada com um único objetivo, a melhora do desempenho. Para se praticar deliberadamente é necessária uma instrução especializada, feedback constante e necessariamente a mesma não ser prazerosa durante sua realização (ERICSSON et al., 1993).

Ericsson et al. (1993) e Ericsson (2005) afirmam existir diversas restrições à prática deliberada que podem exercer influência crítica para 0 alcance do alto desempenho. Estas restrições que influenciam o desempenho do atleta são restrições de motivação, esforço e recursos.

A restrição de motivação é um fator inerente à prática deliberada, pois o prazer imediato pode não ser percebido pelo atleta, que repete centenas de vezes o mesmo movimento durante os treinamentos. No entanto, o prazer é experimentado quando $\mathrm{o}$ atleta consegue atingir uma meta traçada (HODGES et al., 2004). A restrição de esforço se caracteriza pela exigência de altos níveis de concentração e empenho durante os treinamentos. Cabe ao treinador minimizar o exagero, planejando o treinamento com ênfase na qualidade e não na quantidade. Já a restrição de recursos é originada, pois a prática deliberada requer gastos com transporte, competições, treinadores, entre outros fatores que compõem a estrutura necessária para o alto nível.

Hodges et al. (2004) em seu estudo qualitativo com nadadores e triatletas, objetivaram verificar os aspectos mais importantes em relação à prática deliberada. Os resultados apontaram que, os nadadores, pela análise de seus diários, consideravam a prática prazerosa. Já os triatletas, avaliados por questionários, consideravam a mesma extremamente extenuante.

Em Jogos Olímpicos, a natação brasileira é uma das modalidades mais vitoriosas de todos os tempos, se consolidando como uma das modalidades que mais conquistou medalhas para - país. Portanto, este estudo justifica-se, primeiramente, por se tratar de um levantamento histórico, analisando o contexto no qual cada medalhista olímpico se desenvolveu, pois se preocupa em compreender as nuanças específicas de cada realidade encontrada por esses atletas, desde sua iniciação, até o mais alto nível de proficiência.

Por conseguinte, o estudo sobre os melhores atletas de uma determinada modalidade se justifica pelo fato de colaborar para a melhoria daqueles que pretendem aperfeiçoar seu nível de treinamento (STRAUSS; CORBIN, 1990; MORGAN; GIACOBBI JR., 2006). Além disso, a visão da realidade que estes indivíduos têm a respeito de seu desenvolvimento serve como base para a criação de programas de desenvolvimento expert por parte de governos (STOTLAR; WONDERS, 2006; VAEYENS et al., 
2008), de mudança nas metodologias empregadas por parte de treinadores, nos comportamentos dos atletas e na compreensão dos componentes da expert performance (ERICSSON; LEHMANN, 1996).

Portanto, este trabalho teve como objetivo verificar o contexto do desenvolvimento expert dos nadadores medalhistas olímpicos brasileiros.

\section{Método}

Para a realização do presente estudo foi utilizada uma abordagem qualitativa, de natureza retrospectiva, que, de acordo com Thomas et al. (2007), representa uma visão holística do fenômeno (PATTON, 1980; 2002).

\section{Amostra}

A amostra foi composta por oito nadadores experts em suas especificidades que apresentaram, no momento da conquista olímpica, idade de 20,61 \pm 1,52 anos e tempo de prática de 11,37 $\pm 3,29$ anos, além disso, os atletas iniciaram sua prática esportiva com 6,5 \pm 3,29 anos. Para a inclusão na amostra, o critério de seleção foi a conquista de, pelo menos, uma medalha (ouro, prata ou bronze) em qualquer uma das edições dos Jogos Olímpicos, conquistada em provas individuais ou coletivas (revezamentos). Os voluntários tiveram suas identidades resguardadas, aleatoriamente identificados por A1 (atleta 1), A2 (atleta 2), .... e A8 (atleta 8) com a finalidade de manter 0 anonimato dos participantes. Este estudo foi aprovado pelo Comitê de Ética em Pesquisa da Universidade Federal de Minas Gerais sob protocolo número ETIC 195/09.

\section{Instrumentos}

Os instrumentos utilizados foram uma ficha de identificação, com o objetivo de caracterizar a amostra, e um roteiro de entrevista retrospectiva semi-estruturada (PATTON, 1980; 2002; CÔTÉ et al., 2005; JOHNSON et al., 2008).

Os critérios utilizados para estruturar as perguntas do roteiro foram baseados nos modelos de desenvolvimento de experts (BLOOM, 1985; ERICSSON et al., 1993; CSIKSZENTMIHALYI et al., 1993; CÔTÉ et al., 1995). Patton (2002) e Bogdan e Bicklen (1994) afirmam que um dos critérios para se estabelecer uma entrevista é a partir de algum modelo existente na literatura.

Neste tipo de entrevista, os aspectos a serem investigados obedecem aos critérios de relevância estabelecidos pelo pesquisador (PATTON, 1980; 2002; TESCH, 1990; JOHNSON et al., 2008). As perguntas são previamente listadas como roteiro, e podem se desviar do curso inicial, dessa forma, assumem uma característica mais flexível, pois o investigador estabelece a seqüência das mesmas durante a entrevista.

As questões específicas podem ser formuladas para os diferentes participantes de um estudo (PATTON, 1980; TESCH, 1990; CÔTÉ et al., 2005; JOHNSON et al., 2008), pois umas das características neste tipo de pesquisa é a compreensão das realidades múltiplas que determinam o desenvolvimento dos indivíduos (CÔTÉ et al., 2005). Portanto, a partir do supracitado, este estudo contou com uma pergunta inicial conduzida em todas as entrevistas: "Como ocorreu seu desenvolvimento na natação?". Por conseguinte, a entrevista transcorreu de acordo com a análise do contexto do desenvolvimento desses atletas.

Os equipamentos utilizados para realizar as entrevistas foram dois gravadores digitais (Panasonic RR-US450), um notebook (Acer Aspire 3690-2023) e o software para a transcrição dos arquivos de áudio (Voice Editing Premium Edition 2.0).

\section{Procedimento}

Com o objetivo de compreender a viabilidade do estudo, foi realizado, via telefonema, um primeiro contato com os medalhistas olímpicos. Foi enviado a cada voluntário um e-mail explicando os objetivos do estudo, assim como o termo de consentimento livre e esclarecido, que deveria ser assinado e devolvido ao pesquisador no dia da realização da entrevista. As entrevistas foram agendadas de acordo com a disponibilidade dos voluntários. Nas datas agendadas, o pesquisador entrevistou os voluntários em suas respectivas cidades, em locais tranqüilos, onde os voluntários poderiam ficar à vontade para responder às perguntas da entrevista e preencher a ficha de identificação.

A duração média das entrevistas foi de aproximadamente 60 minutos, sendo que as mesmas foram gravadas e transcritas (PATTON, 1980; 2002; CÔTÉ et al., 1995; MARTENS, 1987; THOMAS et al., 2007; JOHNSON et al., 2008). Os dados foram categorizados e passaram pelo expert-rating de três doutores em pesquisa qualitativa para aumentar a credibilidade/legitimidade dos dados (MATHINSON, 1988).

\section{Análise dos dados}

As narrativas foram categorizadas e analisadas por meio de análise de conteúdo (Mini Unidades - MUs) (CÔTÉ, et al., 1993; JOHNSON, et al., 2008; FERREIRA, 2010a). Uma Mini Unidade (MU) representa uma parte, que pode ser uma linha, parágrafo ou mais de um parágrafo do corpo de um texto, que exemplifica uma idéia 
expressada pelo pesquisador de forma clara e objetiva. Neste estudo, as narrativas foram categorizadas e sub categorizadas de acordo com as similaridades identificadas entre as diversas MUs estabelecidas.

Após a avaliação dos experts em pesquisa qualitativa, as MUs foram subdivididas em categorias e sub categorias estabelecidas como representativas dos fatores investigados. Para a análise, as gravações resultaram em 253 páginas de texto que produziram $473 \mathrm{MUs}$, onde $218 \mathrm{MUs}$ foram selecionadas, gerando um grupo de quatro categorias, nove subcategorias e nove propriedades (Figura 1), distribuídos dedutivamente para análise e discussão (STRAUSS; CORBIN, 1990).

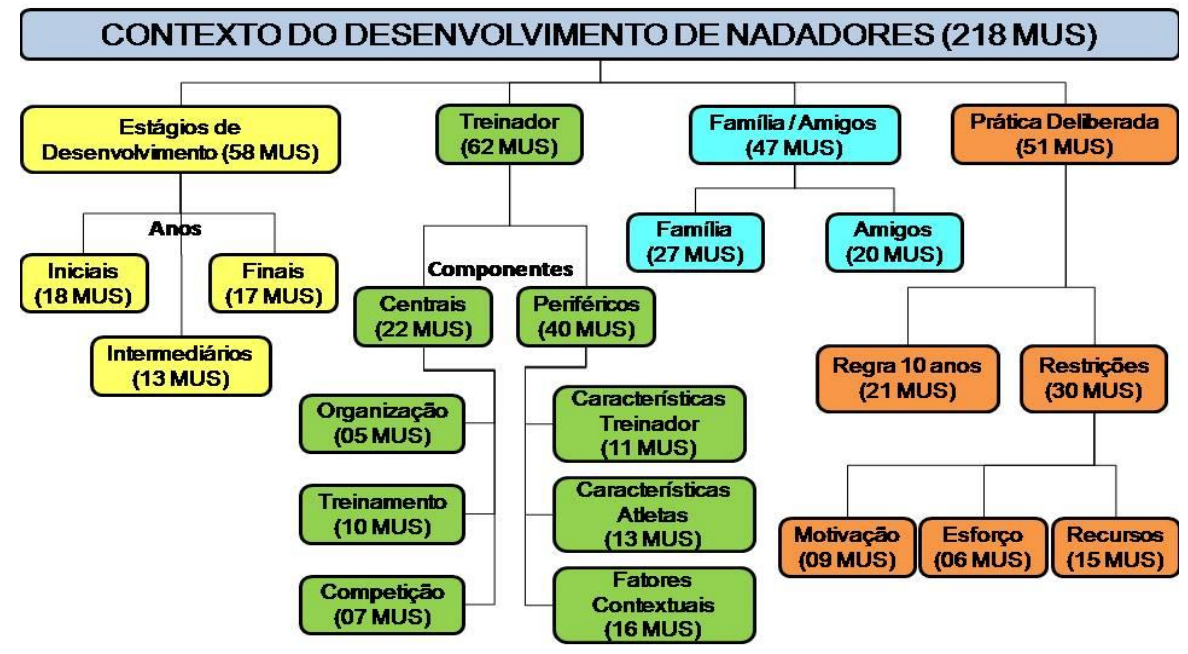

Figura 1- Estrutura das MUs do desenvolvimento dos nadadores medalhistas olímpicos brasileiros.

\section{Resultados e Discussão}

Como já exposto, o objetivo deste trabalho foi verificar o contexto do desenvolvimento expert dos nadadores medalhistas olímpicos brasileiros. Dessa forma, a apresentação e discussão dos resultados seguiram a ótica do contexto do desenvolvimento, ou seja, os estágios de desenvolvimento, o papel do treinador, a presença da família / amigos e a relação com a prática deliberada. Algumas MUs foram utilizadas para ilustrar os resultados.

\section{Estágios de desenvolvimento - Início da carreira esportiva}

Em relação aos estágios de desenvolvimento, foi estabelecido o primeiro estágio como o foco da discussão neste estudo, já que fatores determinantes à iniciação são fundamentais para a progressão do desempenho do atleta.

Tabela 1- Motivos que levaram ao início da prática esportiva dos medalhistas olímpicos

\begin{tabular}{cc}
\hline Atletas & Início da prática (motivos para iniciação) \\
\hline A1 & Desenvolvimento muscular \\
A2 & Irmãos atletas (Influência da família) \\
A3 & Prática de vários esportes (influência da família) \\
A4 & Afogamento \\
A5 & Influência da família \\
A6 & Irmãos atletas (Influência da família) \\
A7 & Problemas respiratórios/Influência da família \\
A8 & Influência da família \\
\hline
\end{tabular}

Identificar os principais motivos para o início da prática esportiva (Tabela 1) é determinante para compreender o tipo de envolvimento que a criança poderá vir a ter com a modalidade esportiva. 
Os resultados indicam que a qualidade do apoio recebido, desde o motivo inicial para inserção na modalidade, é um dos fatores preponderantes para o progresso do atleta. Tal fator é destacado como crítico por Moraes, Rabelo e Salmela (2004) no decorrer do processo de aprendizagem, pois este estágio é fundamental para o desenvolvimento de valores, identidade esportiva, motivação e envolvimento a longo prazo no esporte (CÔTÉ; HAY, 2002).

Seis atletas (A2, A3, A5, A6, A7, A8) apresentaram como principal motivo para iniciação esportiva, uma influência direta da família. Já os atletas A1 e A4 tiveram um motivo primário que desencadeou uma influência indireta da família. Contudo, a família foi, direta ou indiretamente, responsável pela inserção e manutenção do atleta no esporte, disponibilizando apoio emocional e financeiro para que não ocorra - abandono precoce da modalidade, 0 que corrobora Bloom (1985) e Falk et al. (2004). Tal influência pode ser ilustrada pela MU a seguir.

A2 - "Bem, o meu envolvimento aconteceu porque eu venho de uma família de nadadores, meus três irmãos nadaram, eu sou o mais novo. Meu pai é um entusiasta em todos os esportes, principalmente na natação. Começou com meu irmão mais velho que era asmático e o médico sugeriu que ele fizesse natação. Aí meu pai pôs - A. na natação, ele se envolveu, gostou demais. Depois veio o R., veio o R., depois veio eu."

Para Côté (1999), Côté et al. (2003) e Côté et al. (2009), no primeiro estágio, a inserção da criança em diversas modalidades é de suma importância, pois desenvolve 0 gosto pela atividade aprimorando sua experiência psicomotora, e posteriormente se especializa em um único esporte. Esta relação é exemplificada pela MU do atleta $\mathrm{A} 3$.

A3 - "Eu comecei no T. quando tinha cinco pra seis anos, eu participava de uma escola de ginástica, até uma ginástica generalizada nada específica da natação, com vários esportes... E gostei! Sempre me ambientei bem na água, me senti bem e acho que o desenvolvimento foi relativamente rápido porque tive facilidade de aprender alguns estilos..."

\section{Treinador}

O treinador assume papel fundamental para a continuidade da carreira do atleta, pois é responsável pelo aprimoramento da técnica e por empregar e desenvolver estratégias de motivação. As principais características dos treinadores mais marcantes se encontram na tabela 2.

Tabela 2- Principais características dos treinadores mais marcantes

\begin{tabular}{c|c|c|c|c}
\hline Atletas & \multicolumn{4}{c}{ Características dos treinadores } \\
\hline & 10 Treinador & 20 Treinador & 3o Treinador & 4-Treinador \\
\hline A1 & Disciplinador & Técnico & Detalhista & - \\
A2 & Motivador & "Cientista da Natação" & - & - \\
A3 & Motivador & Expert & - & - \\
A4 & Motivador & Técnico & "Cientista da Natação" & Expert \\
A5 & Seriedade & Motivador & Expert \\
A6 & Disciplinador & Profissionalismo & & - \\
A7 & Manager ("paizão") & & Profissionalismo & - \\
A8 & & Motivador & Disciplinador & Expert
\end{tabular}

Os atletas se referiram a um treinador de cada estágio de desenvolvimento. Algumas características encontradas em cinco dos oito treinadores (A2, A3, A4, A7 e A8), referentes ao primeiro estágio, merecem destaque, pois esses indivíduos são caracterizados como carismáticos, motivadores e brincalhões, o que corrobora a literatura que afirma que a principal função do treinador é ser um motivador da criança (BLOOM, 1985; CÔTÉ, 1999; MORAES; RABELO; SALMELA, 2004; MORAES; SALMELA; RABELO; VIANA JUNIOR, 2004; CÔTÉ et al., 2009,
FERREIRA et al., 2010). A MU abaixo ilustra tal característica.

A8 - "Meu primeiro treinador foi o M. S., eu acho que a grande jogada dele foi fazer a natação ser uma diversão para mim. Realmente começar como uma brincadeira. Aquela coisa do grupo, de ter um monte de amigos, de fazer o grito de guerra."

Seis atletas (A2, A3, A4, A5, A6 e A8) no decorrer de suas carreiras, buscaram por treinadores mais experientes e especializados no treinamento de alto rendimento, objetivando a melhora do desempenho, o que resultou em alguns casos, com as mudanças para outros 
centros de treinamentos, confirmando Bloom (1985) e Côté (1999). Abaixo é destacada a narrativa do atleta $A 8$ que elucida o supracitado.

A8 - "...quando eu fui para os EUA... aí sim, foi um negócio completamente diferente de tudo que eu já tinha visto na vida. O B. fazia treino na hora... aquela coisa de você se concentrar, aquela dificuldade de você atingir o objetivo no treino... O grande lance são os desafios que ele solta antes do treino."

Martindale et al. (2007) avaliaram, por meio de uma entrevista semi-estruturada, 16 treinadores britânicos de várias modalidades esportivas. Os resultados indicaram que para o desenvolvimento máximo dos atletas, os treinadores devem estabelecer critérios que encorajem suas equipes, visando a excelência, o que corrobora os estudos de Helsen et al. (1998), Feltz et al., (1999) e Moraes, Salmela, Rabelo e Viana Junior (2004). $\mathrm{O}$ atleta $\mathrm{A} 7 \mathrm{se}$ refere, a partir da MU a seguir, ao seu treinador como um gerenciador da sua carreira.

A7 - “...eu convivi muito com o S. S. que foi
meu treinador na olimpíada, eu posso falar que
era um paizão para mim. Ele fazia o
planejamento de treinamento, ele conversava
com o psicólogo, conversava com nutricionista,
conversava com o restante da comissão técnica
na verdade, então o único trabalho que eu tinha
na verdade era de treinar, eu encontrava tudo
pronto."

\section{Família / Amigos}

O questionamento realizado para conhecer 0 tipo de apoio recebido pelos atletas foi por intermédio da pergunta: "Qual a influência da sua família e de seus amigos durante sua carreira esportiva?"

O tipo do apoio proporcionado pela família (Tabela 3) é um dos norteadores da manutenção do indivíduo na modalidade esportiva.

Tabela 3- Tipo de apoio recebido pelo atleta durante sua carreira esportiva, advindo da família

\begin{tabular}{c|ccc}
\hline \multirow{2}{*}{ Atletas } & \multicolumn{3}{|c}{ Apoio da família durante a carreira } \\
\cline { 2 - 4 } & Início & Meio & Ápice \\
\hline A1 & Financeiro/Motivação & Financeiro/Motivação & Financeiro/Motivação \\
A2 & Financeiro/Motivação & Financeiro/Motivação & Motivação \\
A3 & Financeiro/Motivação & Financeiro/Motivação & Motivação \\
A4 & Financeiro/Motivação & Financeiro/Motivação & - \\
A5 & Financeiro/Motivação & Financeiro/Motivação & Motivação \\
A6 & Financeiro/Motivação & Motivação & Motivação \\
A7 & Financeiro/Motivação & Motivação & Motivação \\
A8 & Financeiro/Motivação & Financeiro/Motivação & Gerenciamento/Motivação \\
\hline
\end{tabular}

O tipo de apoio ofertado pelos pais no início e durante a carreira dos atletas influiu, diretamente, na permanência da criança no esporte. Este apoio que se manteve para todos os nadadores se reflete na motivação para permanecer na modalidade, assim como no comprometimento com a carreira e a prática, o que corrobora os estudos de Vernacchia et al. (2000), Durand-Bush e Salmela (2002), Salmela e Moraes (2003) e Mallet e Hanrahan (2004), já que eles transmitem segurança a partir de incentivos financeiros e motivacionais, o que é confirmado pela narrativa a seguir.

A8 - “... quando eu vim para São Paulo e fui para os EUA,... todo o cenário atrás que ninguém vê, como escolher o melhor ponto para morar, para estar perto do clube... eu nunca corri atrás, foi sempre meu pai e minha mãe que fizeram tudo. Eu só chegava e tinha que nadar mesmo. "

Para Bruner et al. (2008) e McCarthy e Jones (2007) o desenvolvimento dos atletas, ao longo de suas carreiras, ocorre por intermédio do bom relacionamento com seus amigos, o que corrobora, em parte, os resultados encontrados no estudo. Para os atletas A2, A3, A5 e A8, os amigos são fundamentais para, na infância, irem aos treinamentos (envolvimento social) e, posteriormente, para injetarem motivação, já que todo o vínculo social destes indivíduos advém do ambiente esportivo. A MU do atleta A2 demonstra a importância de tal relação.

A2 - “... sem dúvida são os amigos que você
faz na natação que realmente te norteiam, que
faz com que você dê conta de estar ali,
treinando, passando aquelas 4 horas que
realmente são muito sacrificantes... essa
amizade que te dá essa força. Se não fosse
isso, eu acho que a natação nem existiria. Eu
lembro que quando eu era menino, eu ia nadar
por causa dos meninos, da amizade. Eu
simplesmente estava ali porque eu gostava do
ambiente, meus amigos todos estavam lá, eu
fazia parte de um grupo."

Baker e Horton (2004) apontam que as principais influências que os atletas têm para a realização da excelência esportiva são a família e 
os amigos. A família, através de um apoio emocional e financeiro e os amigos por meio de um apoio emocional e competitivo. Samulski et al. (2009) encontraram resultados similares ao avaliarem a transição da carreira de atletas de alto rendimento. Estes resultados corroboram com os encontrados neste estudo, já que a família e os amigos são peças indissolúveis no processo do desenvolvimento atlético destes nadadores.
A prática deliberada é um dos fatores que compõem a excelência de um indivíduo em determinado campo. Dessa forma, determinar o scout do treinamento (Tabela 4), ou seja, saber o volume do treinamento por meio da avaliação da metragem, do número de sessões e da quantidade de horas despendidas com 0 treinamento é uma das formas de quantificar tal prática.

\section{Prática deliberada}

Tabela 4- Dias, sessões, horas e quilômetros treinados

\begin{tabular}{c|c|cccc}
\hline \multirow{2}{*}{ Atletas } & \multicolumn{5}{|c}{ Prática Deliberada } \\
\cline { 2 - 5 } & Dias/semana & Sessões/dia & Horas de treinamento/dia & Km/dia \\
\hline A1 & 6 & 1 a 2 & 4 & 6 a 8 \\
A2 & 6 & 1 a 3 & 8 & 8 a 24 \\
A3 & 6 & 1 a 2 & 4 a 5 & 12 a 20 \\
A4 & 6 & 2 & 4 a 6 & 16 a 20 \\
A5 & 6 & 2 & 5 & 14 a 18 \\
A6 & 6 & 1 a 2 & 6 a 8 & 10 a 20 \\
A7 & 6 & 2 & 6 & 8 a 15 \\
A8 & 6 & 1 a 2 & 2 & 4 a 8 \\
\hline${ }^{*}$ Média anual & & &
\end{tabular}

Os atletas praticaram em média 9,1 anos para atingirem o alto nível, o que se aproxima da regra dos 10 anos de Ericsson et al. (1993) que são confirmadas em consonância com outros estudos (ERICSSON; RORING; NANDAGOPAL, 2007; WARD et al., 2007; COLVIN, 2007; WILLIAMS; ERICSSON, 2008).

O treinamento foi estruturado e desenvolvido por meio de práticas extenuantes (Tabela 4) confirmando Baker e Davids (2007), que citam que o sucesso atingido está associado a uma melhor estrutura de treinamento. Altas cargas de treinamentos podem promover adaptações nos níveis de força (JONES; RUTHERFORD, 1987), na capacidade aeróbia e anaeróbia (HELGERUD et al., 2001) e nas habilidades mentais (DURANDBUSH et al., 2001), como pode ser observada, segundo a narrativa do atleta $A 2$.

A2 - "Então eu cheguei a treinar 24 mil metros por dia, três treinos de oito mil... sempre treinei fundo, depois passei para meio fundo. Então, minha principal característica é suportar o limiar de dor muito grande."

Observa-se na Tabela 4, que a média de volume de treinamento diário $(\mathrm{km} / \mathrm{dia})$ apresenta uma variação, que pode ser justificada pela implantação de diferentes métodos de treinamento, ou pela elaboração específica dos mesmos, elucidado pelas seguintes MUs.

A4 - "Então, o S. foi muito importante porque me deu uma experiência grande de limite de treinamento. Cheguei a treinar $20 \mathrm{~km}$ por dia... Passar seis horas na piscina, coisa assim de doido."

A8 - "O máximo que chegou foi 3.900 metros/sessão. É o aquecimento bem de leve para entrar na série, a série principal, soltou e vai embora.... a gente estava nadando 20 Km/semana."

No entanto, para se atingir a excelência, o indivíduo se depara com algumas restrições à prática deliberada (recursos, esforço e motivação) que podem ser determinantes para se atingir o alto nível. Estas restrições (Tabela 5) podem prejudicar o desenvolvimento do atleta durante um planejamento plurianual.

Tabela 5- As dificuldades, restrições, encontradas pelos atletas durante suas carreiras

\begin{tabular}{c|lcc}
\hline Atletas & \multicolumn{3}{c}{ Restrições } \\
\hline A1 & Motivação & Financeira & Recursos \\
A2 & Saudade & Tempo (clima) & - \\
A3 & Motivação & Fisiológica & - \\
A4 & Motivação & - & - \\
A5 & Motivação & - & - \\
A6 & Motivação & Recursos & - \\
A7 & Motivação & - & - \\
A8 & - & - & -
\end{tabular}

Perdurar no alto rendimento exige do atleta a capacidade de superar as restrições impostas pela prática deliberada. A restrição de motivação 
(falta de prazer imediato com a prática), esforço (exigência nos níveis de concentração e empenho) e recursos (humanos, financeiros) podem levar ao declínio do comprometimento com a modalidade e interferir no rendimento dos atletas (ERICSSON et al., 1993; ERICSSON, 2005).

Os resultados encontrados neste estudo apontam que a principal restrição apontada pelos atletas é a motivacional. Seis dos oito atletas (A1, A3, A4, A5, A6, A7) afirmam que a restrição de motivação é determinante para o declínio do rendimento. A narrativa do atleta A3 ilustra tal restrição.

A3 - "Acho que o principal era o cansaço, principalmente porque no tipo de treinamento que era aplicado antigamente o desgaste emocional era muito maior, as distâncias que se nadavam eram muito maiores. Então você tinha o cansaço físico e o emocional."

Sendo assim, a partir do apresentado, tudo indica que os motivos que levaram ao início da prática esportiva, a presença de treinadores especializados, as influências ao longo das carreiras e o tipo de prática favoreceram o desenvolvimento dos atletas. Dessa forma, foram encontrados fatores pertinentes a todas as realidades avaliadas.

\section{Conclusão}

A partir do objetivo proposto e da discussão dos resultados apresentada, concluiu-se que 0 contexto do desenvolvimento composto pela iniciação à prática, relação com os treinadores, influência da família / amigos e a prática deliberada, contribuiu de forma fundamental para o desenvolvimento dos atletas. As realidades múltiplas apontam que não existe necessariamente um padrão restrito de excelência, indicando ainda que a expertise pode ser alcançada por meio de diversos contextos.

O início da carreira dos atletas é de fundamental importância para seu desenvolvimento posterior. A família esteve presente direta ou indiretamente, influenciando a criança a iniciar e permanecer na natação. A ocorrência mais comum foi a iniciação da prática pela influência de irmãos e/ou pais atletas.

As principais características dos treinadores são a habilidade dos primeiros treinadores têm em transformar a prática em diversão para os atletas (prazer), a seriedade e a disciplina que alguns treinadores ensinaram aos atletas durante os estágios subseqüentes ao primeiro e a excelência e o conhecimento aprofundado do esporte, principal característica dos treinadores responsáveis pela conquista da medalha olímpica.

A presença da família é fundamental para o desenvolvimento do nadador, pois com apoio financeiro e motivacional a permanência da criança no esporte é alcançada. Assim, ao longo da carreira do nadador, a família assume um papel mais motivacional. Os amigos podem assumir um papel positivo nos treinamentos por meio do convívio social no ambiente esportivo.

A superação das restrições da prática deliberada e o treinamento altamente organizado possibilitaram o máximo desempenho nas competições. As cargas de treinamento variaram devido à implantação de diferentes métodos de treinamento e pela especificidade de cada atleta.

Este estudo limitou-se a analisar o contexto do desenvolvimento de nadadores medalhistas olímpicos brasileiros, a partir de uma análise histórica de cada contexto em que estes atletas estavam inseridos. Sugerem-se novos estudos que avaliem o contexto de desenvolvimento no qual o mesmo grupo de atletas esteja inserido, assim como uma análise histórica em outras modalidades esportivas.

\section{Referências}

BAKER, J.; DAVIDS, K. Sound and fury, signifying nothing?: future directions in the nature-nurture debate. International Journal of Sport

Psychology, Roma, v. 38, n. 1, p. 135-143, 2007.

BAKER, J.; HORTON, S. A review of primary and secondary influences on sport expertise. High Ability Studies, London, v. 15, n. 2, p. 211-228, 2004. Disponível em: $<$ http://dx.doi.org/10.1080/1359813042000314781 >. Acesso em: 06 out. 2010.

BAKER, J.; HORTON, S.; ROBERTSONWILSON, J.; WALL, M. Nurturing sport expertise: factors influencing the development of elite athlete. Journal of Sports Science and Medicine, Burlington, v. 2, n. 1, p. 1-9, 2003.

BENGOECHEA, E. G.; STREAN, W. B.; WILLIAMS, D. J. Understanding and promoting fun in youth sport: coaches' perspectives. Physical Education and Sport Pedagogy, London, v. 9, n. 2, p. 197-214, 2004. Disponível em:<http://dx.doi.org/10.1080/1740898042000294 994>. Acesso em: 06 out. 2010.

\section{BLOOM, B. S. Developing talent in young} people. New York: Ballantine Books, 1985. 
BOGDAN, R.; BICKLEN, S. Investigação

qualitativa em educação. Portugal: Porto, 1994.

BRUNER, M. W.; MUNROE-CHANDLER, K. J.;

SPINK, K. S. Entry into the elite sport: a preliminary investigation into the transition experiences of rookie athletes. Journal of Applied Sport Psychology, London, v. 20, n. 2, p. 236-252, 2008. Disponível em: $<$ http://dx.doi.org/10.1080/10413200701867745>. Acesso em: 06 out. 2010.

COLVIN, G. What it takes to be great. American Swimming Magazine, Sedona, v. 2, n. 1, p. 1013, 2007.

CÔTÉ, J. The influence of the family in the development of talent in sport. The Sport Psychologist, Champaign, v. 13, n. 4, p. 395417, 1999.

CÔTÉ, J.; HAY, J. Children's involvement in sport: a developmental analysis. In: SILVA, J. M.; STEVENS, D. (Org.). Psychological foundations of sport. Boston: Allyn and Bacon, 2002. p. 484-502.

CÔTÉ, J.; BAKER, J.; ABERNETHY, B. From play to practice: A developmental framework for the acquisition of expertise in team sport. In: STARKES, J.; ERICSSON, K. A. (Org.). Recent advances in research on sport expertise. Champaign: Human Kinetics, 2003. p. 89-114.

CÔTÉ, J.; ERICSSON, K. A.; LAW, M. P. Tracing the development of athletes using retrospective interview methods: a proposed interview and validation procedure for reported information. Journal of Applied Sport Psychology, London, v. 17, n. 1, p. 1-19, 2005. Disponível em: <http://dx.doi.org/10.1080/10413200590907531>. Acesso em: 06 out. 2010.

CÔTÉ, J.; LIDOR, R.; HACKFORT, D. ISSP position stand: to sample or to specialize?: seven postulates about youth sport activities that lead to continued participation and elite performance. The International Journal of Sport and Exercise Psychology, London, v. 9, n. 1, p. 7-17, 2009.

CÔTÉ, J.; SALMELA, J. H.; BARIA, A.; RUSSELL, S. J. Organizing and interpreting unstructured qualitative data. The Sport Psychologist, Champaign, v. 7, n. 2, p. 127-137, 1993.

CÔTÉ, J.; SALMELA, J. H.; TRUDEL, P.; BARIA, A.; RUSSEL, S. J. The coaching model: a grounded assessment of expert gymnastic coaches' knowledge. Journal of Sport \& Exercise Psychology, v. 17, n. 1, p. 1-17, 1995.
CSIKSZENTMIHALYI, M.; RATHUNDE, K.; WHALEN, S. Talent teenagers: the root of success and failure. Cambridge: Cambridge University Press, 1993.

DURAND-BUSH, N.; SALMELA, J. H.; GREENDEMERS, I. The Ottowa mental skills assessment tool (OMSAT-3*). The Sport Psychologist, Champaign, v. 15, n. 1, p. 1-19, 2001.

DURAND-BUSH, N.; SALMELA, J. H. The development and maintenance of expert athletic performance: perceptions of world and olympic champions. Journal of Applied Sport Psychology, London, v. 14, n. 3, p. 154-171, 2002. Disponível em:

$<$ http://dx.doi.org/10.1080/10413200290103473>. Acesso em: 06 out. 2010.

ERICSSON, K. A. Recent advances in expertise research: a commentary on the contributions to the special issue. Applied Cognitive

Psychology, San Francisco, v. 19, n. 2, p. 233241, 2005. Disponível em: $<$ http://dx.doi.org/10.1002/acp.1111>. Acesso em: 06 out. 2010.

ERICSSON, K. A.; LEHMANN, A. C. Expert and exceptional performance: evidence of maximal adaptation to task constraints. Annual Reviews Psychology, Palo Alto, v. 47, p. 273-305, 1996.

ERICSSON, K. A.; KRAMPE, R. T.; TESCHROMER, $C$. The role of deliberate practice in the acquisition of expert performance. Psychological Review, Washington, v. 100, n. 3, p. 363-406, 1993.

ERICSSON, K. A.; PRIETULA, M. J.; COKELY, E. $T$. The making of an expert. Harvard Business Review, Cambridge, v. 85, n. 7-8, p. 18-26, 2007. Disponível em: $<$ http://dx.doi.org/10.1225/R0707J>. Acesso em: 06 out. 2010.

ERICSSON, K. A.; RORING, R. W.; NANDAGOPAL, K. Giftedness and evidence for reproducibly superior performance: an account based on the expert performance framework. High Ability Studies, London, v. 18, n. 1, p. 3-56, 2007. Disponível em:

$<$ http://dx.doi.org/10.1080/13598130701350593>. Acesso em: 06 out. 2010.

ERICSSON, K. A.; WHYTE, J.; WARD, P. Expert performance in nursing: reviewing research on expertise in nursing within the framework of the expert performance approach. Advances in Nursing Science, Philadelphia, v. 30, n. 1, p. 5871, 2007. 
FALK, B.; LIDOR, R.; LANDER, Y.; LANG, B.

Talent identification and early development of elite water-polo players: a 2-year follow-up study.

Journal of Sports Sciences, v. 22, n. 4, p. 347-

355, 2004. Disponível em:

$<$ http://dx.doi.org/10.1080/0264041031000164156

6r>. Acesso em: 06 out. 2010.

FELTZ, D.; CHASE, M.; MORITZ, S.; PHILLIP, S. A conceptual model of coaching efficacy: preliminary investigation and instrument development. Journal of Educational

Psychology, Baltimore, v. 91, n. 4, p. 765-776, 1999.

\section{FERREIRA, R. M. O contexto de}

desenvolvimento de nadadores medalhistas olímpicos brasileiros. 2010. 121f. Dissertação (Mestrado em Treinamento Esportivo) - Escola de Educação Física, Fisioterapia e Terapia Ocupacional da Universidade Federal de Minas Gerais, Belo Horizonte, 2010a.

FERREIRA, R. M. O Contexto do desenvolvimento de nadadores medalhistas olímpicos brasileiros. Motriz, Rio Claro, v. 16, n. 3, p. 810-810, 2010b.

FERREIRA, R. M.; PENNA, E. M.; MORAES, L. C. C. A. Características fundamentais de treinadores de nadadores medalhistas olímpicos brasileiros. In: CONGRESSO BRASILEIRO, 14; CONGRESSO

INTERNACIONAL DE PSICOLOGIA DO ESPORTE, 7, 2010, Curitiba. Anais... Curitiba: [S.n.], 2010. v. 1. Disponível em: <http://www.crppr.org.br/congressoesporte/pdf pa inel/15.pdf >. Acesso em: 06 out. 2010.

GOULD, D.; EKLUND, R. C.; JACKSON, S. Coping strategies used by U.S. Olympic Wrestlers. Research Quarterly for Exercise and Sport, Reston, v. 64, n. 1, p. 83-93, 1993.

GUSTAFSSON, H.; KENTTA, G.; HASSMÉN, P.; LUNDQVIST, C. Prevalence of burnout in competitive adolescent athletes. The Sport Psychologist, Champaign, v. 21, n. 1, p. 21-37, 2007.

HAYS, K.; MAYNARD, I.; THOMAS, O.; BAWDEN, M. Sources and types of confidence identified by world class sport performers. Journal of Applied Sport Psychology, v. 19, n. 4, p. 434456, 2007. Disponível em: <http://dx.doi.org/10.1080/10413200701599173>. Acesso em: 06 out. 2010.

HELGERUD, J.; ENGEN, L. C.; WISLOFF, U.; HOFF, J. Aerobic endurance training improves soccer performance. Medicine \& Science in
Sports \& Exercise, Philadelphia, v. 33, n. 11, p. 1925-1931, 2001.

HELSEN, W. F.; STARKES, J. L.; HODGES, N. J. Team sports and the theory of deliberate practice. Journal of Sport and Exercise Psychology, Champaign, v. 20, n. 1, p. 12-34, 1998.

HODGES, N. J.; STARKES, J. L.; KERR, T.; WEIR, P. L.; NANANIDOU, A. Predicting performance times from deliberate practice hours for triathletes and swimmers: what, when, and where is practice important? Journal of

Experimental Psychology: Applied, Washington, v. 10, n. 4, p. 219-237, 2004. Disponível em: <http://dx.doi.org/10.1037/1076-898X.10.4.219>. Acesso em: 06 out. 2010.

HOLT, N. L.; DUNN, J. G. H. Toward a grounded theory of the psychosocial competencies and environmental conditions associated with soccer success. Journal of Applied Sport Psychology, London, v. 16, n. 3, p. 199-219, 2004. Disponível em:

$<$ http://dx.doi.org/10.1080/10413200490437949>. Acesso em: 06 out. 2010.

JOHNSON, M. B.; CASTILLO, Y.; SACKS, D. N.; CAVAZOS JUNIOR, J.; EDMONDS, W. A.; TENENBAUM, G. Hard work beats talent until talent decides to work hard: coaches' perspectives regarding differentiating elite and non-elite swimmers. International Journal of Sports Science \& Coaching, Brentwood, v. 3, n. 3, p. 417-430, 2008.

JONES, D. A.; RUTHERFORD, O. M. Human muscle strength training: the effects of three different regimens and the nature of the resultant changes. The Journal of Psychology,

Philadelphia, v. 391, n. 1, p. 1-11, 1987.

LEHMANN, A. C.; ERICSSON, K. A. The historical development of domains of expertise:

performance standards and innovations in music. In: STEPTOE, A. (Org.). Genius and the mind. Oxford: Oxford University Press, 1998. p. 67-94.

MALLET, C. J.; HANRAHAN, S. J. Elite athletes: why does the 'fire' burn so brightly. Psychology of Sport and Exercise, Burlington, v. 5, n. 2, p. 183-200, 2004. Disponível em: $<$ http://dx.doi.org/10.1016/S1469-0292(02)000432>. SALMELA, J. H.; RABELO, A. S.; VIANA JUNIOR

MARTENS, R. Science, knowledge, and sport psychology. The Sport Psychologist, Champaign, v. 1, n. 1, p. 29-55, 1987.

MARTINDALE, R. J. J.; COLLINS, D.; DAUBNEY, J. Talent development: a guide for practice and 
research within sport. Quest, Champaign, v. 57, n. 4, p. 353-375, 2005.

MARTINDALE, R. J. J.; COLLINS, D.; ABRAHAM, A. Effective talent development: the elite coach perspective in UK sport. Journal of Applied Sport Psychology, London, v. 19, n. 2, p. $187-$ 206, 2007. Disponível em: <http://dx.doi.org/10.1080/10413200701188944>. Acesso em: 06 out. 2010.

MATHINSON, S. Why Triangulate?. Educational Researcher, Washington, v. 17, n. 2, p. 13-17, 1988.

MCCARTHY, P. J.; JONES, M. V. A qualitative study of sport enjoyment in the sampling years. The Sport Psychologist, Champaign, v. 21, n. 4, p. 400-416, 2007.

MORAES, L. C. C. A.; SALMELA, J. H. Expertise no esporte. In: GARCIA, E. S.; LEMOS, K. L. M. (Org.). Temas Atuais em Educação Física e Esportes. Belo Horizonte: Health, 2003. p. 159172. v. 7.

MORAES, L. C. C. A.; SOUSA, C. D. A. As diferentes influências da tríade pais-atletastreinadores na trajetória de desenvolvimento de judocas brasileiros de nível internacional. In: GARCIA, E. S.; LEMOS, K. L. M. (Org.). Temas Atuais em Educação Física e Esportes. Belo Horizonte: Gráfica Silveira, 2004. p. 111-132. v. 8.

MORAES, L. C. C. A.; RABELO, A. S.; SALMELA, J. H. Papel dos pais no desenvolvimento de jovens futebolistas. Psicologia: reflexão e crítica, Porto Alegre, v. 17, n. 2, p. 211-222, 2004.

MORAES, L. C. C. A.; SALMELA, J. H.; RABELO, A. S.; VIANA JUNIOR, N. S. Le rôle des parents dans le développement des jeunes joueurs de football et de tennis brésiliens. Revue International des Sciences du Sport et de I'Education Physique-Staps, Montpellier, v. 25, n. 64, p. 109-126, 2004.

MORGAN, T. K.; GIACOBBI JUNIOR., P. R. Toward two grounded theories of the talent development and social support process of highly successful collegiate athletes. The Sport Psychologist, Champaign, v. 20, n. 3, p. 295313, 2006.

PATTON, M. Q. Qualitative evaluation methods. Beverly Hills: Sage, 1980.

PATTON, M. Q. Qualitative evaluation methods. 3 ed. Califórnia: Sage, 2002.
PLANT, E. A.; ERICSSON, K. A.; HILL, L.; ASBERG, K. Why study time does not predict grade point average across college students: Implications of deliberate practice for academic performance. Contemporary Educational Psychology, Burlington, v. 30, n. 1, p. 96-116, 2005. Disponível em: $<$ http://dx.doi.org/10.1016/i.cedpsych.2004.06.001 >. Acesso em: 06 out. 2010.

SALMELA, J. H.; MORAES, L. C. C. A. Development of Expertise: the role of coaching, families and cultural contexts. In: STARKES, J. L.; ERICSSON, A. K. (Org.). Expert performance in sports. Champaign: Human Kinetics, 2003. p. 272-291.

SAMULSKI, D. M.; MORAES, L. C.; FERREIRA, R. M.; MARQUES, M. P.; SILVA, L. A.; LÔBO, I. L.; MATOS, F. O.; SANTIAGO, M. L. M.;

FERREIRA, C. H. S. Análise das transições das carreiras de ex-atletas de alto nível. Motriz, Rio Claro, v. 15, n. 2, p. 310-317, 2009.

SIMON, H. A.; CHASE, W. G. The mind's eye in chess. In: CHASE, W. G. (Org.). Visual information processing. San Diego: Academic Press, 1973. p. 215-281.

SINGER, R. N.; JANELLE, C. M. Determining sport expertise: from genes to supremes. International Journal of Sport Psychology, Roma, v. 30, n. 2, p. 117-150, 1999.

STOTLAR, D. K.; WONDERS, A. Developing elite athletes: a content analysis of US national governing body systems. International Journal of Applied Sports Sciences, Roma, v. 18, n. 2, p. 121-144, 2006.

STRAUSS, A.; CORBIN, J. Basics of qualitative research: grounded theory procedures and techniques. Newbury Park: Stage, 1990.

TESCH, R. Qualitative Research: analysis types and software tools. New York: Falmer Press, 1990.

THOMAS, J. R.; NELSON, J. K.; SILVERMAN, S. J. Métodos de pesquisa em atividade física. 5 . ed. Porto Alegre: Artmed, 2007.

VAEYENS, R.; LENOIR, M.; WILLIAMS, A. M.; PHILIPPAERTS, R. M. Talent identification and development programmes in sport. Sports Medicine, Baltimore, v. 9, n. 38, p. 703-714, 2008.

VERNACCHIA, R. A.; MCGUIRE, R. T.; REARDON, J. P.; TEMPLIN, D. P. Psychosocial characteristics of olympic track and field athletes. 
International Journal of Sport Psychology,

Roma, v. 31, n. 1, p. 5-23, 2000.

WARD, P.; HODGES, N. J.; STARKES, J. L.;

WILLIAMS, A. M. The road to excellence:

deliberate practice and the development of

expertise. High Ability Studies, London, v. 18, n.

2, p. 119-153, 2007. Disponível em:

<http://dx.doi.org/10.1080/13598130701709715>.

Acesso em: 06 out. 2010.

WILLIAMS, A. M.; ERICSSON, K. A. From the guest editors: how do experts learn? Journal of

Sport \& Exercise Psychology, Champaign, v.

30, n. 6, p. 653-662, 2008.

Este estudo contou com o apoio da Fapemig (Fundação de Amparo à Pesquisa do estado e Minas Gerais) e da CBDA (Confederação Brasileira de Desportos Aquáticos).

\section{Endereço:}

Renato Melo Ferreira

Av. Presidente Carlos Luz, 4664, Pampulha

Belo Horizonte MG Brasil

31310-250

Telefone e Fax: (31) 3409-2325

E-mail: renato.mf@hotmail.com

Recebido em: 02 de novembro de 2010.

Aceito em: 18 de setembro de 2011.

\section{(c) (7)}

Motriz. Revista de Educação Física. UNESP, Rio Claro, SP, Brasil - elSSN: 1980-6574 - está licenciada sob Creative Commons - Atribuição 3.0 